\title{
Preventive therapy for asthma in children; a 9-year experience in Eastern Finland
}

\author{
M. Korppi, L. Kuikka, K. Remes
}

Preventive therapy for asthma in children; a 9-year experience in Eastern Finland. $M$. Korppi, L. Kuikka, K. Remes. CERS Journals Ltd 1995.

ABSTRACT: The long-term treatment modalities of bronchial asthma were studied in children from a defined Finnish population from 1985 to 1993, with special reference to changes during the study period.

The data on maintenance drugs in children with asthma from five years $(1985$, 1987, 1989, 1991 and 1993) were retrospectively retrieved from the computerized registers. The reliability of the data for the diagnosis and basic treatment of asthma was checked by one of the authors, who compared the data with the patient cards from the hospital.

The number of children with doctor-diagnosed asthma increased continuously during the surveillance period. The proportion of children receiving preventive medication increased concomitantly; this increase was most pronounced between 1987 and 1989. The most common preventive drug was sodium cromoglycate; its use increased from $14 \%$ in 1985 to $58 \%$ in 1993 . The use of inhaled steroids remained stable at $17-19 \%$ in all surveillance years.

Our treatment policy is in accordance with the international consensus statement published in 1989; however, the change towards preventive medication occurred before its publication.

Eur Respir J., 1995, 8, 1318-1320.

The prevalence and severity of childhood asthma are on the increase $[1,2]$. Asthma deaths are also increasing among children in industrialized countries [3, 4]. For these reasons, an international meeting was held in 1988, during which a consensus statement was adopted for the diagnosis and therapy of bronchial asthma in children [1]. After its publication in 1989, the protocol was evaluated by Finnish paediatricians and paediatric allergists, and it was also adopted for use in Finland. The consensus statement was re-evaluated in 1991, when the treatment protocols were revised to a more simplified form [5].

Maintenance medication, including sodium cromoglycate and inhalation corticosteroids, as well as slow-release theophylline given perorally, have long been used in children with severe asthma [6]. During the last $10 \mathrm{yrs}$, before there was any widely accepted consensus, a clear trend was evident: long-term therapy has been started in younger children and in earlier phases of the disease [1]. The trend has been similar, although not evaluated or published, in Finland. Therefore, we retrospectively analyzed Finnish data containing the occurrence and maintenance medication of bronchial asthma in children during five separate years (1985, 1987, 1989, 1991 and 1993).

The aim of this study was to evaluate the changes that took place in the long-term treatment modalities, with special reference to changes before and after the consensus statement published in 1989.
Dept of Paediatrics, Kuopio University Hospital, Kuopio, Finland.

Correspondence: M. Korppi Kuopio University Hospital Dept of Paediatrics SF-70211 Kuopio Finland

Keywords: Asthma prevention beclomethasone bronchial asthma budesonide indication therapy sodium cromoglycate

Received; June 151994 Accepted after revision April 131995

\section{Material and methods}

The Department of Paediatrics, of the Kuopio University Hospital, provides care to all paediatric patients requiring hospital treatment from a geographically defined area. In this area, the province of Kuopio, the population under the age of $15 \mathrm{yrs}$ is about 53,000. The only out-patient clinic for asthmatic children functions in the university hospital under the supervision of a paediatric allergist. This unit provides diagnostic and consultation services for children with mild to moderate asthma; those patients with severe asthma also receive primary treatment at this unit. Thus, the paediatric allergist (KR) of our hospital and her staff are responsible, either directly or indirectly, for the diagnosis and long-term therapy of most asthmatic children living in the whole province.

On every visit, the basic data, including the long-term therapy (sodium cromoglycate or nedocromil, inhaled corticosteroid, such as budesonide or beclomethasone, slow-release theophylline or some combination of these drugs) are recorded into a computerized register. According to our treatment policy the patients, whether or not they are receiving preventive medication, use beta - $_{2}$-sympathomimetics, salbutamol or terbutaline, during symptomatic phases of the disease; these drugs are not used as long-term treatment. For the present evaluation, the data on maintenance treatment from five years $(1985,1987$, 
Table 1. - Number of children with doctor-diagnosed bronchial asthma in two age groups during the five year study

\begin{tabular}{|c|c|c|c|c|c|c|}
\hline \multicolumn{7}{|c|}{ Children with asthma } \\
\hline \multirow{3}{*}{$\frac{\begin{array}{l}\text { Year } \\
\mathrm{n}\end{array}}{1985}$} & \multirow{3}{*}{$\begin{array}{c}\begin{array}{c}\text { All ages } \\
\text { n }\end{array} \\
273\end{array}$} & \multirow{2}{*}{\multicolumn{2}{|c|}{$\begin{array}{c}\leq 5 \mathrm{yrs} \text { old } \\
\%\end{array}$}} & \multirow{3}{*}{$\begin{array}{c}\text { p-value } \\
\text { NS }\end{array}$} & \multicolumn{2}{|c|}{$>5$ yrs old } \\
\hline & & & & & $\mathrm{n}$ & $\%$ \\
\hline & & 79 & 29 & & 194 & 71 \\
\hline 1987 & 367 & 95 & 26 & NS & 272 & 74 \\
\hline 1989 & 346 & 65 & 19 & NS & 281 & 81 \\
\hline 1991 & 515 & 118 & 23 & 0.001 & 394 & 77 \\
\hline 1993 & 636 & 250 & 39 & & 386 & 61 \\
\hline
\end{tabular}

p-values evaluate the changes in the occurences of bronchial asthma in different years in the study population $\leq 5 \mathrm{yrs}$ of age.

1989, 1991 and 1993) were retrieved from the computerized register. In every case, the reliability of the data was checked by one author (KR), who compared the data with the patient cards of our hospital. In this way, the equivalence of the diagnosis of bronchial asthma, and the adequacy of asthma therapy during the five study years could also be confirmed.

The Chi-squared test with a correction devised by Yates was used for statistical analysis of the data.

\section{Results}

As seen in table 1, the number of children with bronchial asthma increased continuously from 1985 to 1993 . The age distribution of patients was quite stable up to the year $1991 ; 19-29 \%$ of the children were less than 5 yrs of age. In 1993, however, the proportion of these young children increased to $39 \%$.

The distribution of the treatment modalities is presented in figure 1 . The use of beta $a_{2}$-sympathomimetics as sole medication for asthmatic children clearly decreased during the surveillance period. This decrease was most prominent between 1987 and 1989. Correspondingly, the use of cromoglycate increased. The use of inhaled steroids, beclomethasone or budesonide, remained quite stable during the whole surveillance period. Only a small minority of children were receiving either slow-release theophylline or combinations.

\section{Discussion}

According to the international consensus statement, adopted in 1988, published in 1989 and introduced in Finland in 1989-1990, cromoglycate is the drug of choice for preventive medication in mild or moderate asthma; inhaled steroids are used in severe asthma and in cases resistant to cromoglycate therapy [1, 5]. From 1988 onwards, the majority of our asthmatic children have been receiving preventive medication, and cromoglycate has been the most frequently used drug. In 1993, as many as $58 \%$ of the asthmatic children used cromoglycate (or nedocromil) as their basic medication. Less than $20 \%$ of our patients were treated with inhaled steroids; unlike other reports, this proportion has not increased [7]. None of our patients had used systemic steroids as long-term therapy.

Asthmatic children wheezing only occasionally may use sympathomimetics intermittently if they are symptomless with normal physical activity between the acute episodes $[1,5,7]$. Less than $20 \%$ of our children were on this treatment modality in 1993 . The figure was $60 \%$ in $1985,57 \%$ in 1987 , and $30 \%$ in 1989 . Thus, a new approach, emphasizing the role of preventive therapy, took place in 1988. Preventive medication was introduced at an earlier phase of the disease and for younger patients [7].

The number of asthmatic children in our area has increased continuously; it doubled from 1985 to 1993 . The occurrence of asthma increased especially in children

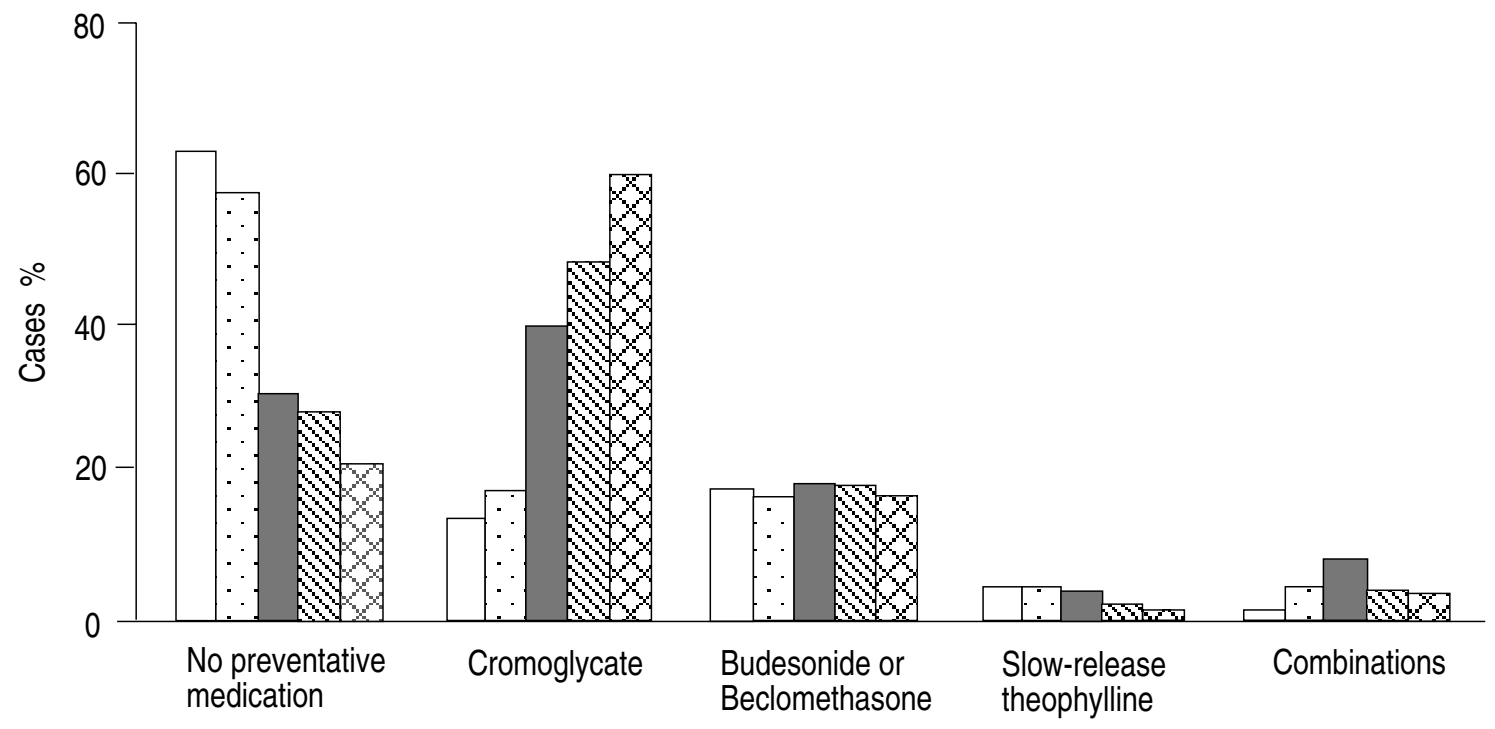

Fig. 1. - Distribution of long-term treatment modalities of bronchial asthma in children from a defined child population during five years $(1985,1987,1989,1991$ and 1993). Children on nedocromil therapy are included in the cromoglycate group. $\square$ : $1985 ; \square: 1987 ; \square: 1989 ;$ : $\square$ :1991; $\square: 1993$. 
younger than 5 yrs old. This could possibly reflect earlier referral and earlier diagnosis of asthma [8]. However, a more probable explanation is that the real increase of asthma prevalence has taken place in accordance with observations from many other countries $[2,7]$.

There are two main reasons for the increased use of inhaled steroids in maintenance therapy of bronchial asthma. Asthma has become more severe and more chronic than previously [2], and inflammation is now considered to be the primary event in the pathogenesis of asthma [9]. Recently, inhaled steroids have also been suggested as the first-line therapy in children with mild-to-moderate asthma [10]. However, these steroids have many potential side-effects, including adrenal suppression and effects on growth $[11,12]$. On the other hand, chronic asthma if treated insufficiently retards growth and delays puberty [13].

In summary, a clear change towards preventive medication of bronchial asthma in children took place in 1988 in our area. Our results suggest that it is possible to restrict the use of steroids in childhood to a selected minority of patients. When a child really needs inhaled steroids, the lowest, sufficiently effective dose should be used [11]. Cromoglycate (or nedocromil) is a safe alternative with no side-effects [14]; based on our experience they alone constitute suitable medication for over half of asthmatic children.

\section{References}

1. Warner JO, Götz M, Landau LJ, et al. Management of asthma: a consensus statement. Arch Dis Child 1989; 64: $1065-1102$.
2. Burr ML, Butland BK, King S, Vaughan-Williams E. Changes in asthma prevalence: two surveys 15 years apart. Arch Dis Child 1989; 64: 1452-1456.

3. Burney P. Asthma deaths in England and Wales 1931-1985: evidence for a true increase in asthma mortality. $J$ Epidemiol Commun Health 1988; 42: 316-320.

4. Bousquet J, Hatton F, Godard P, Michel FB. Asthma mortality in France. J Allergy Clin Immunol 1987; 80: 389-394.

5. Warner JO, Neijens HJ, Landau LI, et al. Asthma: a follow-up statement from an international paediatric asthma consensus group. Arch Dis Child 1992; 67: 240248.

6. Weinberger M. Antiasthmatic therapy in children. Clin Pediatr North Am 1989; 36: 1251-1284.

7. Carlsen K-H. Epidemiology of childhood asthma. Eur Respir Rev 1994; 4(17): 5-9.

8. Andersson H. Increase in hospital admissions for childhood asthma: trends in referral, severity, and readmissions from 1970 to 1985 , in a health region of United Kingdom. Thorax 1989; 44: 614-619.

9. Bousquet J, Chanez P, Champbell A, et al. Inflammatory processes in asthma. Int Arch Allergy Appl Immunol 1991; 91: 227-232.

10. Agertoft L, Pedersen S. Effects of long-term treatment with an inhaled corticosteroid on growth and pulmonary function in asthmatic children. Respir Med 1994; 88: 373-381.

11. Boner A, Piacentini G. Inhaled corticosteroids in children. Is there a safe dosage? Drug Safety 1993; 9; 9-20.

12. Pedersen S. Safety aspects of corticosteroids in children. Eur Respir Rev 1994; 4(17): 33-43.

13. Balfour-Lynn L. Growth and childhood asthma. Arch Dis Child 1986; 61: 1049-1055.

14. Price J. Corticosteroids and other anti-inflammatory agents in the treatment of children. Eur Respir Rev 1994; 4(17): 27-32. 